An experimental study of the dynamic properties of nanoparticle colloids with identical magnetization but different particle size

P.C. Fannin, C.N. Marin, K. Raj, C. Couper, P. Barvinschi

PII: S0304-8853(12)00158-8

DOI: $\quad$ doi:10.1016/j.jmmm.2012.02.061

Reference: MAGMA57407

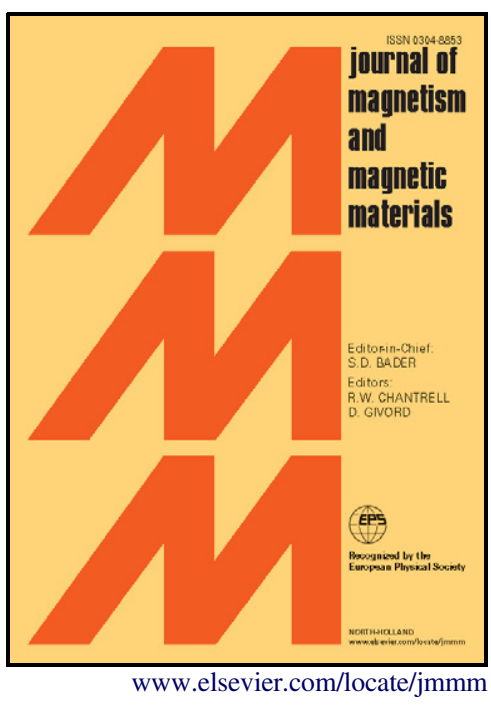

To appear in: Journal of Magnetism and Magnetic Materials

Cite this article as: P.C. Fannin, C.N. Marin, K. Raj, C. Couper and P. Barvinschi, An experimental study of the dynamic properties of nanoparticle colloids with identical magnetization but different particle size, Journal of Magnetism and Magnetic Materials, doi:10.1016/j.jmmm.2012.02.061

This is a PDF file of an unedited manuscript that has been accepted for publication. As a service to our customers we are providing this early version of the manuscript. The manuscript will undergo copyediting, typesetting, and review of the resulting galley proof before it is published in its final citable form. Please note that during the production process errors may be discovered which could affect the content, and all legal disclaimers that apply to the journal pertain. 


\title{
An experimental study of the dynamic properties of nanoparticle colloids with identical magnetization but different particle size

\author{
P. C. Fannin ${ }^{a^{*}}$, C. N. Marin ${ }^{b}$, K. Raj ${ }^{\text {c }}$, C. Couper ${ }^{a}$ and P. Barvinschi ${ }^{b}$ \\ ${ }^{a}$ Department of Electronic and Electrical Engineering, Trinity College, Dublin 2, Ireland \\ ${ }^{b}$ West University of Timisoara, Faculty of Physics, no. 4, V. Parvan Blv., 300223, \\ Timisoara, Romania \\ ${ }^{c}$ Ferrofluidics Corporation, Nashua, NH, USA
}

\begin{abstract}
Measurements of the frequency dependent complex magnetic susceptibility, $\chi(\omega)=\chi^{\prime}(\omega)-i \chi^{\prime \prime}(\omega)$, have been used to determine the dynamic properties of three specially prepared $400 \mathrm{G}(0.04 \mathrm{~T})$ magnetic fluids. The samples, denoted by sample 1 , sample 2 and sample 3, consisted of magnetite particles of mean diameter $6.4 \mathrm{~nm}, 7.5 \mathrm{~nm}$ and $9 \mathrm{~nm}$ respectively and were identical in terms of carrier liquid, surfactant and particle material.

From polarised ferromagnetic measurements, the anisotropy field, $H_{A}$, the Landau-Lifshitz damping parameters, $\alpha$, and the precessional decay time, $\tau_{0}$ of the particles were determined. The results show that the $H_{A}$ value for sample 3 was almost twice that of sample 1 and 2 , thus confirming that the smaller the particle size, the lower the value of $H_{A}$. The damping parameter, $\alpha$, was found to be 0.174 (for sample 1), 0.18 (for sample 2) and 0.16 (for sample 3). The values determined for the precessional decay time, $\tau_{0}$ were $1.197 \cdot 10^{-9} \mathrm{~s}, 1.157 \cdot 10^{-9} \mathrm{~s}$ and $0.789 \cdot 10^{-9} \mathrm{~s}$, for samples 1,2 and 3 respectively.
\end{abstract}

Keywords: Magnetic fluids; Ferrimagnetic resonance; Magnetic anisotropy; Damping parameter; Precessional decay time

PACS: 47.65.Cb - Magnetic fluids; 76.50.+g - Ferrimagnetic resonance; 75.30.Gw Magnetic anisotropy

${ }^{*}$ E-mail: pfannin@tcd.ie 


\section{Introduction}

Magnetic fluids comprise of a distribution of particles having radii ranging from approximately 2 to $10 \mathrm{~nm}$, covered with a surfactant and dispersed in a carrier liquid. The particles are in the single domain state, with magnetic moment, $m$ given by $m=M_{S} v$, where $M_{S}$ denotes the spontaneous magnetization of the bulk material of the particle and $v$ is the magnetic volume of the particle.

Thermal fluctuations of small size particles induces a reduction of effective magnetocrystalline anisotropy field, $H_{A, \text { eff }}$, compared with the bulk value, $H_{A, \text { bulk. }}$. In the presence of a strong polarizing field, $H$ (i.e. larger than the anisotropy field), the theoretically predicted effective value of the uniaxial magnetocrystalline anisotropy field, as measured by magnetic resonance, is given by [1], [2]:

$$
H_{A, \text { eff }}=H_{A, \text { bulk }} \frac{1-3 \xi^{-1} \operatorname{coth}(\xi)+3 \xi^{-2}}{\operatorname{coth}(\xi)-\xi^{-1}}
$$

where $\xi=M_{S} v H / k_{B} T, k_{B}$ is the Boltzmann's constant and $T$ is the temperature of the system.

Studies on nanostructured magnetic recording media regarding the effect of the Landau-Lifshitz damping parameter, $\alpha$, on the switching time (i.e. the time taken by the magnetic moment in overcoming the energy barrier, when a magnetic filed is applied as a write pulse) [3-5] have clearly emphasized the importance of the damping parameter in the magnetic recording process. The ferromagnetic resonance line width of magnetic nanoparticle systems [1] as well as the ratio between the maximum absorption frequency at resonance, $f_{\max }$, and the resonance frequency, $f_{\text {res }},[6]$ are also dependent on $\alpha$. The Néel relaxation process [7] and the intra-well relaxation process [8] are other examples in which $\alpha$ has an important role to play. These few examples are sufficient to highlight the importance of $\alpha$ in the magnetization dynamics of magnetic nanoparticle systems.

As can be observed from Eq. (1), the smaller the particle size, the smaller $H_{A, \text { eff. }}$. Consequently, for small sizes and large temperatures, the magnetic anisotropic nanoparticles tend to behave like isotropic magnetic nanoparticles. The theoretically predicted effective value of $\alpha$ of isotropic magnetic nanoparticles is given by [1]: 


$$
\alpha_{i s o}=\alpha_{0} \frac{\xi-L(\xi)}{\xi L(\xi)}
$$

where $L(\xi)=\operatorname{coth}(\xi)-\xi^{-1}$ is the Langevin function and $\alpha_{0}$ is the limit value of the Landau-Lifshitz damping parameter, which corresponds to very large $\xi$ (strictly speaking, for $\xi \rightarrow \infty$ ). From Eq. (2) one can see that in the case of isotropic magnetic nanoparticles, the smaller the particle size, the larger the effective value of $\alpha$. As the anisotropic small particles approach the behaviour of isotropic particles (see Eq. (1)), one can presume that the damping parameter of anisotropic nanoparticles is particle size dependent.

Another parameter which determines the magnetization dynamics of magnetic nanoparticles is the precessional decay time, $\tau_{0}[1]$,

$$
\tau_{0}=\frac{M_{S}}{2 \alpha \gamma K}=\frac{1}{\alpha \gamma H_{A, e f f}}
$$

where, $\gamma$ is the magneto-mechanical ratio and $K$ is the effective anisotropy constant. As $\alpha$ and $H_{A, \text { eff }}$ are size dependent, $\tau_{0}$ is also expected to be particle size dependent.

The object of this work is to investigate the particle size effect on the parameters of magnetization dynamics (i.e. the measured anisotropy field, $H_{A}$, the Landau-Lifshitz damping parameter, $\alpha$ and the precessional decay time, $\tau_{o}$. The results are also discussed in terms of particle concentration and the possible presence of other iron oxide particles in magnetic fluids.

\section{Complex magnetic susceptibility}

The complex magnetic susceptibility, $\chi(\omega)=\chi^{\prime}(\omega)-i \chi^{\prime \prime}(\omega)$ of an assembly of single domain magnetic particles can be described in terms of its parallel, $\chi_{\|}(\omega)$ and perpendicular, $\chi_{\perp}(\omega)$ components, with [1]: 


$$
\chi(\omega)=\frac{1}{3}\left(\chi_{\|}(\omega)+2 \chi_{\perp}(\omega)\right)
$$

The parallel susceptibility $\chi_{\|}(\omega)$ can be described by the Debye equation [9], with

$$
\chi_{\|}(\omega)=\frac{\chi_{\|}(0)}{1+\mathrm{i} \omega \tau_{\|}}
$$

where $\chi_{\|}(0)$ is the static parallel susceptibility and $\tau_{\|}$is the parallel relaxation time. $\tau_{\|}$ is related to the frequency, $f_{\max , \|,}$, at which the imaginary component of $\chi_{\|}^{\prime \prime}(\omega)$ is a maximum, by the expression $f_{\max , \|}=1 /\left(2 \pi \tau_{\| /}\right)$.

Over the high frequency range considered here, $\tau_{\|}$will be dominated by the Néel relaxation mechanism with relaxation time [1]:

$$
\tau_{\mathrm{N}}=\tau_{\mathrm{D}}(1+2 \sigma 5) \quad \sigma<1
$$

and

$$
\tau_{\mathrm{N}}=\frac{\sqrt{\pi}}{2} \frac{\tau_{\mathrm{D}}}{\sigma^{3 / 2}} \exp (\sigma) \quad \sigma \geq 2
$$

where $\tau_{D}=\tau_{0} \sigma$ is referred to as the rotational diffusion time and $\sigma=K v / k_{B} T$. The perpendicular or transverse susceptibility, $\chi_{\perp}(\omega)$, can have a resonant character; this phenomenon being indicated by a transition in the value of $\chi_{\perp}^{\prime}(\omega)$ from a positive to a negative quantity at a frequency, $f_{\text {res }}$.

The resonance condition for a particle with the uniaxial anisotropy axis oriented at an angle $\theta$ with respect to the polarizing field, in the approximation of no interparticle interaction, is given by [1]:

$$
\omega_{0}=\gamma\left[H+H_{A, b u l k} \frac{L_{2}(\xi)}{L(\xi)} P_{2}(\cos \theta)\right]
$$


where $L_{2}(\xi)=1-3 L(\xi) / \xi$ and $P_{2}$ is the Legendre polynomial.

In a system of magnetic nanoparticles, the anisotropy axes of individual particles may have different orientations with respect to the polarizing field. Also, the particles obey a size distribution and may undergo interparticle interactions and it is possible that apart from the magnetocrystalline anisotropy, other forms of anisotropy may also be present. As a result of averaging, the resonance condition of the magnetic nanoparticle system (Eq. (8)) may be written in the following simplified form:

$$
\omega_{\text {res }}=2 \pi f_{\text {res }}=\gamma\left(H+H_{A}\right)
$$

Here, $H_{A}$ may be expressed as in the case of uniaxial anisotropy, $H_{A}=2 K / M_{S}$, but will include the effects mentioned above.

\section{Experimental result and discussion}

In this work we present measurements of three magnetic fluid samples, namely, sample 1 , sample 2 and sample 3 , consisting of colloidal suspensions of magnetite particles dispersed in a light hydrocarbon oil and having average particle diameter of 6.4 $\mathrm{nm}, 7.5 \mathrm{~nm}$ and $9 \mathrm{~nm}$, respectively. The samples were specially prepared by the manufacturer FerroTec and apart from particle size, the samples were identical in every way (i.e. carrier, surfactant, particle material and fluid magnetisation of $400 \mathrm{G}(0.04 \mathrm{~T})$ ).

In some circumstances, the obtaining process of magnetite nanoparticles for magnetic fluid manufacturing may lead to a mixture of iron oxides, apart from the desired magnetite. In Ref. [10] it is shown that the rate of magnetite formation depends on the $\mathrm{pH}$ value of the reacting medium, the concentration of reagents, the nature of the precipitator and the temperature of synthesis. Most noticeable is $\gamma-\mathrm{Fe}_{2} \mathrm{O}_{3}$ resulting from oxidation of $\mathrm{Fe}_{3} \mathrm{O}_{4}$, but other oxides, like $\mathrm{FeO}$ and $\alpha-\mathrm{Fe}_{2} \mathrm{O}_{3}$ may be obtained together with magnetite.

Both $\mathrm{Fe}_{3} \mathrm{O}_{4}$ and $\gamma-\mathrm{Fe}_{2} \mathrm{O}_{3}$ are magnetically equivalent (meaning that the spontaneous magnetization of magnetite is $4.77 \cdot 10^{5} \mathrm{~A} / \mathrm{m}[11]$ and that of maghemite is $4.17 \cdot 10^{5} \mathrm{~A} / \mathrm{m}$ [12]) and therefore it has not been of much commercially concern in manufacturing magnetic fluids, with respect to their saturation magnetization. 
The presence of "miscellaneous particles" (i.e. other iron oxide particles, different from magnetite particles), can certainly affect the measured properties of magnetic fluids. For instance, in Ref. [13], it was suggested that the results obtained for the particle size distribution were affected by the presence of "miscellaneous particles".

Whilst the spontaneous magnetization of $\mathrm{Fe}_{3} \mathrm{O}_{4}$ and $\gamma-\mathrm{Fe}_{2} \mathrm{O}_{3}$ can have similar values, the same cannot be said with regard to the magneto-crystalline anisotropy constant. The value of the magneto-crystalline anisotropy constant of magnetite (as measured on a single crystal) is $1.1 \cdot 10^{4} \mathrm{Jm}^{-3}$ [14], but the magneto-crystalline anisotropy constant of the cubic phase of maghemite is $4.7 \cdot 10^{3} \mathrm{Jm}^{-3}[15]$ and that of the tetragonal phase of maghemite is of the order of $2.3 \cdot 10^{5} \mathrm{Jm}^{-3}[16]$.

The presence of other iron oxides in the magnetic fluid, apart from magnetite, may lead to experimental values of the anisotropy field which are different from those usually accepted for magnetite nanoparticles in magnetic fluids. For this reason XRD analysis was performed on the magnetic fluid samples. Figure 1 shows the spectra obtained from X-ray analysis of the samples and the diffraction pattern indicates a higher degree of crystallinity of particles in the magnetic fluid samples, displaying the characteristic XRD peaks of magnetite [17], thus indicating the absence of any significant amount of other iron oxides.

\section{Fig.1}

Measurements of the complex magnetic susceptibility, $\chi(\omega)=\chi^{\prime}(\omega)-i \chi^{\prime \prime}(\omega)$, over the frequency range $100 \mathrm{MHz}$ to $6 \mathrm{G} \mathrm{Hz}$, were made by means of the coaxial transmission line technique [18]. For the measurements reported here, a Hewlett-Packard (HP) $50 \Omega$ coaxial line incorporating a coaxial cell with $3 \mathrm{~mm}$ inner diameter and $7 \mathrm{~mm}$ outer diameter was used in conjunction with a HP 8753C network analyzer. The samples were placed in the coaxial cell and standard HP opencircuit $(\mathrm{O} / \mathrm{C})$ and short-circuit $(\mathrm{S} / \mathrm{C})$ test devices used as terminations. Automatic swept measurements of the input impedance of the line containing the samples were measured and from these measurements the complex components, $\chi^{\prime}(\omega)$ and $\chi^{\prime \prime}(\omega)$. were 
determined. The samples were also subjected to 8 values of polarizing field, $H$, over the range 0 and $124 \mathrm{kAm}^{-1}$ and the measurement procedure repeated.

The real, $\chi^{\prime}(\omega)$, and imaginary, $\chi^{\prime \prime}(\omega)$, susceptibility components of the three ferrofluid samples were measured over the stated frequency range and all samples exhibited resonance, as indicated by the $\chi^{\prime}(\omega)$ component changing sign at the frequency, $f_{\text {res }}$, and by the presence of a maximum of the $\chi^{\prime \prime}(\omega)$ component at the frequency $f_{\max }$.

Fig. 2 shows the results obtained in the case of sample 3 (with average particle diameter of $9 \mathrm{~nm}$ ) over the polarising field range 0 and $124 \mathrm{kAm}^{-1}$. These results are in general typical of those obtained for all samples. From this figure it is seen that variation of $H$ over the stated range results in $f_{\text {res }}$ and $f_{\max }$ increasing up to frequencies of $5.66 \mathrm{GHz}$ and $5.5 \mathrm{GHz}$, respectively.

\section{Fig.2}

A plot of $f_{\text {res }}$ against $H$ for all samples is shown in Fig. 3 and as $\omega_{\text {res }}=2 \pi f_{\text {res }}=\gamma\left(H+H_{A}\right)$ the mean value of the anisotropy field, $H_{A}$, and the magnetomechanical ratio, $\gamma$, are determined from the intercept and from the slope of the linear dependence. The results are presented in Table 1.

\section{Fig.3}

Apart from the nature of the material of particles, the effective anisotropy field is affected by various causes such as:

a) the particle concentration (namely, increasing the particle concentration leads to an increase of the anisotropy field [19]);

b) the particle size (namely, increasing the particle size leads to an increase of the anisotropy field [1]); 
c) the particle agglomeration (namely, the presence of the particle agglomeration leads to a larger anisotropy field in comparison with the case of no particle agglomeration [20]);

d) the dispersion of the orientation of anisotropy axes (namely, narrowing the dispersion of the orientation of anisotropy axes leads to the increase of the anisotropy field [21]).

Consequently, taking into account the magnetic dipolar interparticle interactions and after averaging over all orientations of the anisotropy axes and over all orientations of the dipolar field, the resonance frequency may be expressed as [22]:

$$
f_{\text {res }}=\frac{\gamma}{2 \pi}\left(H+a \frac{m}{d_{\text {mean }}^{3}}+b H_{A, e f f}\right)
$$

Here, $d_{\text {mean }}$ is the mean distance between the particles, the parameter $a$ depends on the local structure of the system, whilst the parameter $b$ depends on the orientation of the anisotropy axes.

As can be seen in Table 1, the anisotropy field of sample 3 (with particles having an average diameter of $9 \mathrm{~nm}$ ) is the largest one. In the case of samples 1 and 2, even though they have different average particle diameters, the anisotropy field is approximately the same.

Due to the fact that the samples have the same saturation magnetization ( $4 \pi M_{\infty}=400 G$ ), sample 1 (with smaller particles) will have a larger particle concentration than sample 2. This means that the decrease of the anisotropy field due to the decrease of particle size (in sample 1 with respect to sample 2) is compensated for, by the increase of particle concentration. This leads to approximately the same values of the anisotropy field in samples 1 and $2\left(H_{A} \cong 20 \mathrm{kA} / \mathrm{m}\right)$. However, the effective anisotropy field has different dependencies on each of the above mentioned parameters. This means that the decrease of particle size may not compensate for the increase of particle concentration in any ranges of particle size and particle concentration.

On the other hand, sample 3 has large particles and therefore particle agglomeration is more probable in this sample with respect to the other two, leading to an 
increase of the effective anisotropy field [20]. Moreover, in a given magnetic field, the larger the particle sizes, the narrow the dispersion of the orientation of the anisotropy axes [21]; leading also to an increase of the anisotropy field of sample 3 with respect to the sample 1 and sample 2 .

Inspection of equation (3) indicates that by obtaining one further parameter, $\alpha$, (the Landau-Lifshitz damping parameter), the precessional decay time, $\tau_{0}$, may be evaluated. Fortunately this is a simple matter since, as was reported in Ref. [6], a value of $\alpha$ may be obtained from the plot of $f_{\max } / f_{\text {res }}$ against polarising field, $H$. It has been shown that in the region of strong polarizing fields, $\left(H>H_{A}\right)$,

$$
\alpha=\sqrt{\frac{1-\left(f_{\max } / f_{\text {res }}\right)^{2}}{1+\left(f_{\max } / f_{\text {res }}\right)^{2}}}
$$

Fig.4 shows a plot of $f_{\text {max }} / f_{\text {res }}$ against the polarising field, $H$, for sample 3 , and this plot tends to a constant value of 0.975 , for $H>H_{A}$, resulting in a value of $\alpha=0.16$. By means of the same procedure, the $\alpha$ values of the other two samples were determined. The results are presented in Table 1.

\section{Fig.4}

As shown in Ref. [6], the ratio $f_{\text {max }} / f_{\text {res }}$ depends on the particle concentration and thus the larger the particle concentration, the larger the damping parameter. On the other hand, Eq.(1) predicts that the damping parameter decreases with decreasing particle size. Our samples have the same magnetization, but particles with different average sizes. From one sample to another, the average size increases, but the particle concentration decreases, leading thus to a non-monotonic dependence of the damping parameter on the size of the particles as observed in Table 1.

If in Eq. (3) the measured value, $H_{A}$, of the anisotropy field is used instead of the effective magnetocrystalline anisotropy field, $H_{A, \text { eff }}$, then the resulting precessional decay time, $\tau_{0}$, will include the averaging effect over the orientations of individual anisotropy axes of particles, and the interparticle interaction effect as well as the effect of other 
forms of anisotropy. From the equation, $\tau_{0}=\left(\alpha \gamma H_{A}\right)^{-1}$, the corresponding $\tau_{0}$ values of the samples were computed and the results are given in Table 1.

Table 1

\section{Conclusions}

Measurements of the frequency dependent complex magnetic susceptibility $\chi(\omega)=\chi^{\prime}(\omega)-i \chi^{\prime \prime}(\omega)$, of three specially prepared $400 \mathrm{G}(0.04 \mathrm{~T})$ magnetic nanoparticle colloidal suspensions have been presented. The samples, denoted by sample 1 , sample 2 and sample 3, consisted of magnetite particles of mean diameter $6.4 \mathrm{~nm}, 7.5 \mathrm{~nm}$ and $9 \mathrm{~nm}$ respectively and were identical in terms of carrier liquid, surfactant and particle material.

From polarised, ferromagnetic resonant measurements, the anisotropy field, $H_{A}$, the Landau-Lifshitz damping parameter, $\alpha$, and the precessional decay time, $\tau_{0}$, of the particles were determined.

It has been demonstrated that the anisotropy field, $H_{A}$ is, particle size dependent with the value of $H_{A}$ for sample $3(9 \mathrm{~nm})$ being almost twice that of samples 1 and 2 , thus confirming that the smaller the particle size, the lower the value of $H_{A}$. The anisotropy field of samples 1 and 2 was found to have the same value, even though these samples have different mean particle size $(6.6 \mathrm{~nm}$ for sample 1 and $7.5 \mathrm{~nm}$ for sample 2). As the samples have different particle size and the same magnetization, they have different particle concentration. Therefore, the decrease of the anisotropy field due to the decrease of particle size (in sample 1 with respect to sample 2) is compensated for, by the increase of particle concentration, leading to approximately the same values of the anisotropy field in samples 1 and 2 .

The possibility of the presence of "miscellaneous particles" in the samples [10, 13], which could influence the value of $H_{A}$ was also investigated by means of X-ray analysis. This analysis has confirmed the absence of any significant amount of other iron oxides in the samples thereby indicating that, in this case, the value of $H_{A}$ was not influenced by the presence of other particles types.

As regards the damping parameter, $\alpha$, it was found that sample 3 has a value of 0.16 compared to values of 0.174 and 0.18 for samples 1 and 2, thereby demonstrating that the smaller the particle size, the larger the effective value of $\alpha$. Similarly in the case 
of the precessional decay time, $\tau_{0}$, the values determined were found to be in the generally accepted time region of $10^{-9} \mathrm{~s}$, with samples, 1,2 and 3 having values of $1.197 \cdot 10^{-9} \mathrm{~s}, 1.157 \cdot 10^{-9} \mathrm{~s}$ and $0.789 \cdot 10^{-9} \mathrm{~s}$, respectively. Also in comparing the $\tau_{0}$ value of sample 3 (which has the highest mean particle diameter and the lowest packing fraction) with those of samples 1 and 2, (which have a higher packing fraction, but smaller mean particle diameter), one can conclude that the packing fraction and the particle diameter do influence the precessional decay time, $\tau_{0}$.

\section{Acknowledgements}

P.C.Fannin acknowledges ESA for part funding of this work whilst C.N.Marin acknowledges support of CNMP grant no. 1-32155/2008.

\section{References}

[1] Yu.L.Raikher, M.I.Shliomis, The effective field method in the orientational kinetics of magnetic fluids and liquid crystals, Adv.Chem.Phys. 87, ch.8, (1994) 595.

[2] R. S. de Biasi, T. C. Devezas, J. Appl. Phys. 49 (1978) 2466.

[3] L. He, W.D. Doyle, J. Appl. Phys. 79 (1996) 6489.

[4] M. Bauer, R. Lopusnik, F. Fassbender, B. Hillebrands, J. Magn. Magn. Mater. 218 (2000) 165.

[5] G. Bertotti, I.D. Mayergoyz, C. Serpico, M. Dimian, J. Appl. Phys. 93 (2003) 6909.

[6] P. C. Fannin, C. N. Marin, I. Malaescu, , J. Phys.: Condensed Matter 15 (2003) 4739.

[7] W. F. Brown, Phys. Rev. 130 (1963) 1677.

[8] Yu. P. Kalmykov, W. T. Coffey, S. V. Titov, J. Magn. Magn. Mater. 265 (2003) 44.

[9] P. Debye, Polar Molecules, The Chemical Catalog Company, New York, 1929.

[10] N.M. Gribanov, E.E. Bibik, O.V. Buzunov, V.N. Naumov, J. Magn. Magn. Mater., 85 (1990) 7.

[11] E. Kneller, Ferromagnetismus, Springer-Verlag, Berlin 1962.

[12] R. Hergt, R. Hiergeist, I. Hilger, W. A. Kaiser, Y. Lapatnikov, S. Margel and U.

Richter, J. Magn. Magn. Mater. 270 (2004) 345.

[13] M. M. Maiorov, E. Blums, K. Raj, Physics Procedia, 9 (2010) 74.

[14] L. R. Bickford, Phys. Rev. 78 (1950) 449. 
[15] G. Bate, Magnetic Oxides, ed. D. J. Craik, Wiley, London 1975.

[16] P. Dutta, A. Manivannan, M. S. Seehra, N. Shah and G. P. Huffman, Phys. Rev. B 70 (2004) 174428.

[17] JCPDS - International Centre for Diffraction Data, PDF-2 Data Base (1999).

[18] P. C. Fannin, C. MacOireachtaigh and C. Couper, J. Magn. Magn. Mater., 322 (2010) 2428.

[19] P. C. Fannin, I. Mălăescu, C. N. Marin, J. Magn. Magn. Mater., 289 (2005) 162.

[20] C. N. Marin, J. Magn. Magn. Mater., 250 (2002) 197.

[21] Yu. L. Raikher, V. I. Stepanov, Phys. Rev. B, 50 (1994) 6250.

[22] P.C.Fannin, C.N.Marin, V.Socoliuc, G.M.Istrătucă, A.T.Giannitsis, J.Phys.D:

Appl.Phys., 36 (2003) 1227. 


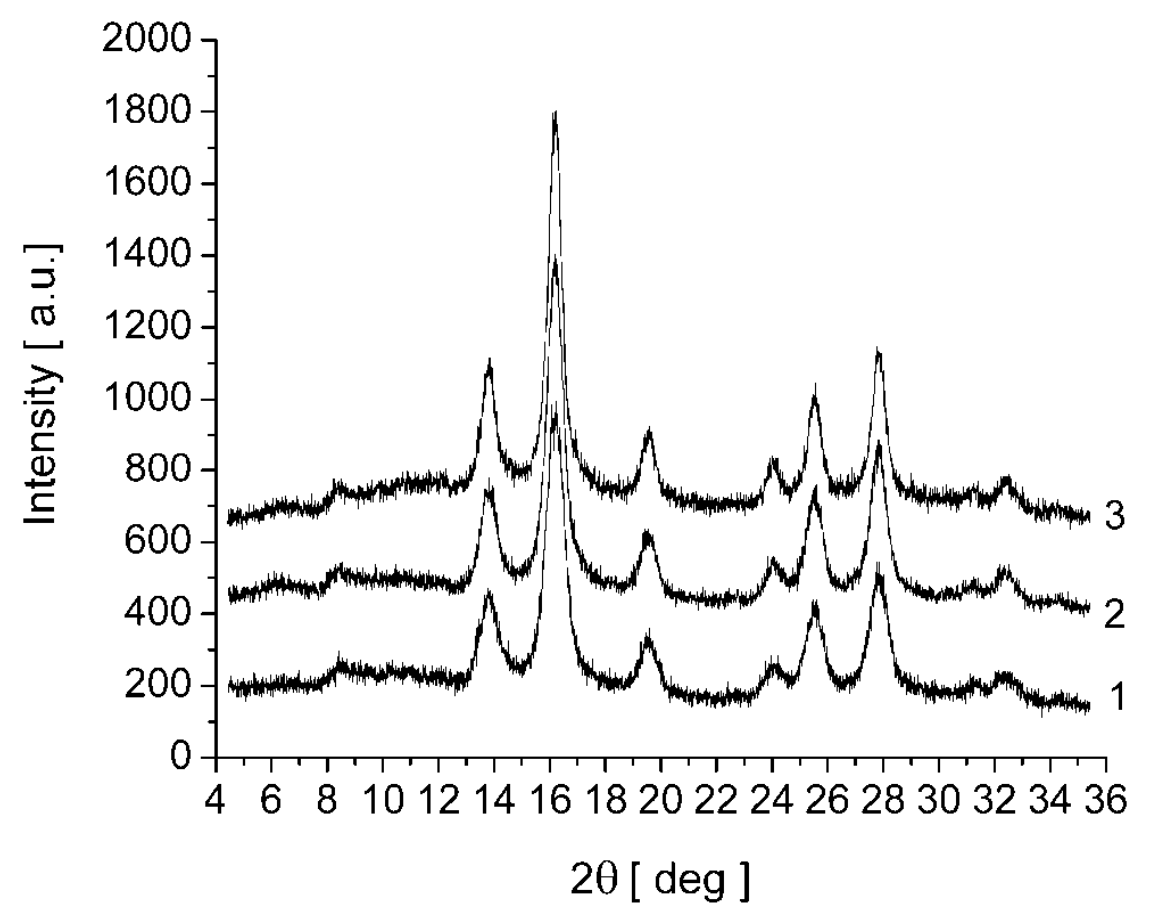

Figure 1

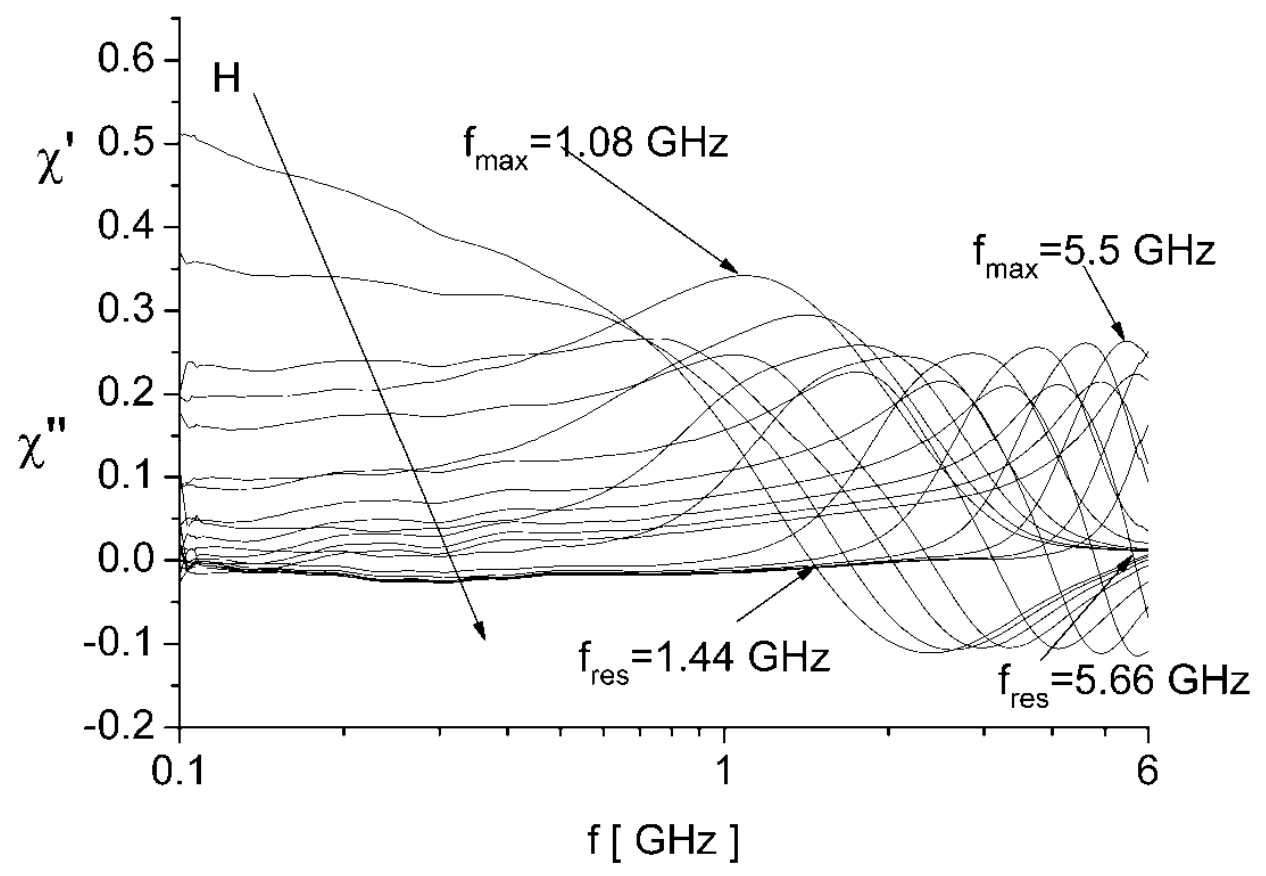

Figure 2 


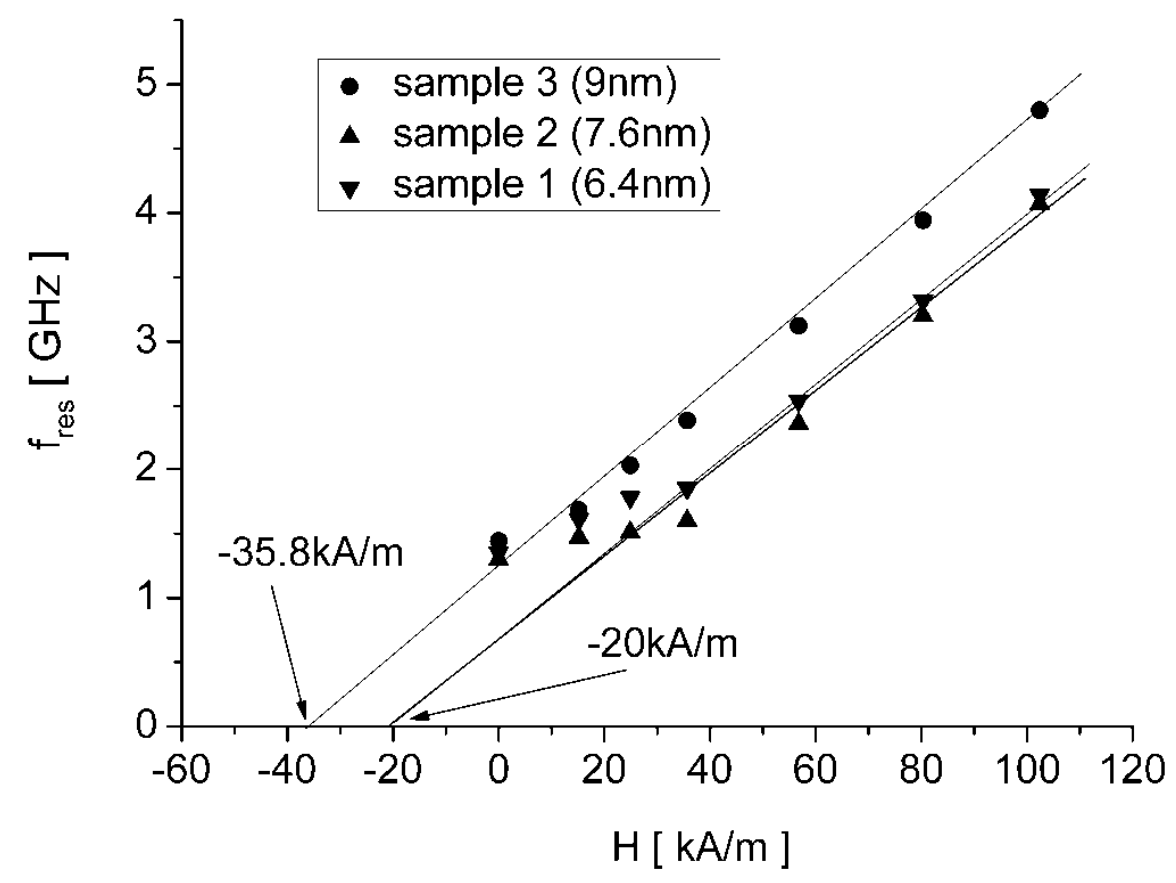

Figure 3

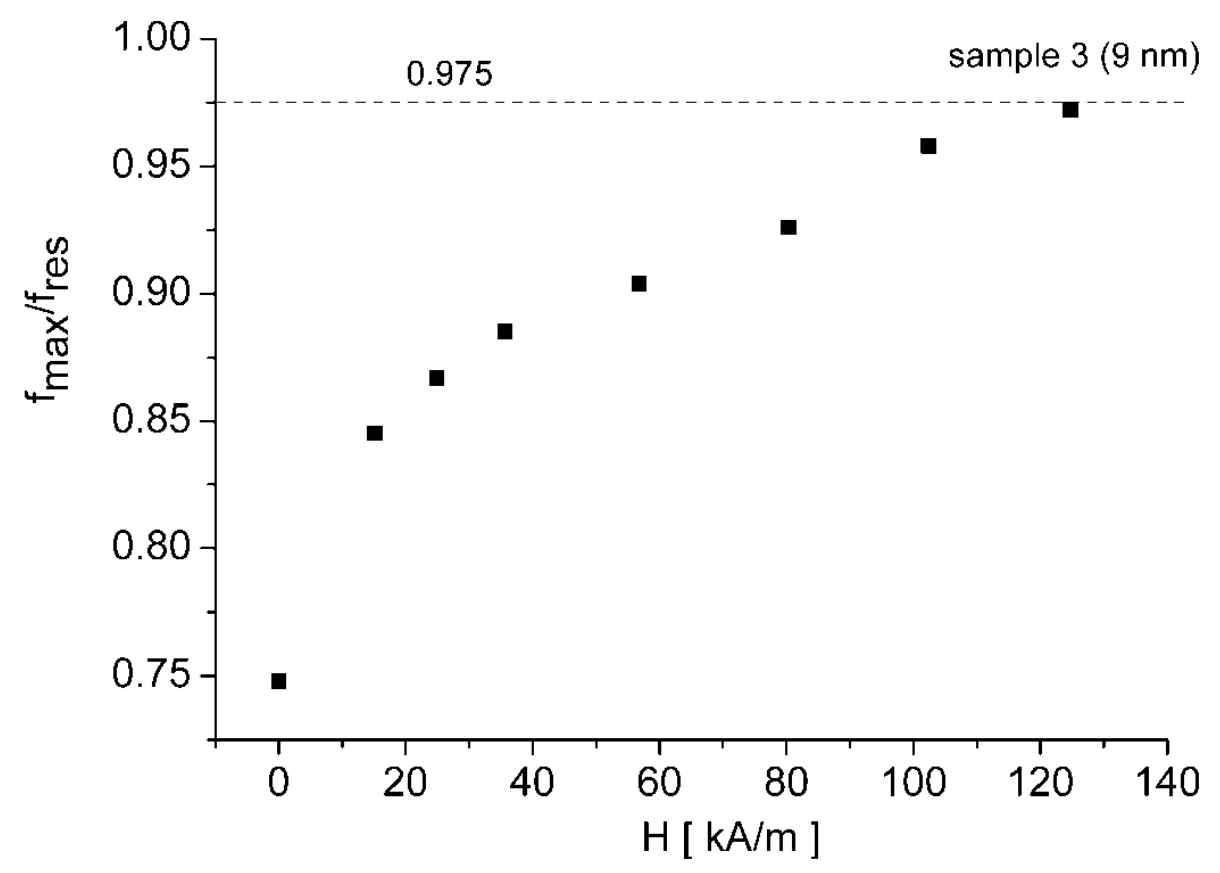

Figure 4 


\section{Figure captions}

Fig.1. Mo-K $\alpha$ X-ray diffraction patterns of magnetic fluid samples: 1 - sample 1 (6.4 $\mathrm{nm}) ; 2$ - sample $2(7.5 \mathrm{~nm})$ and 3 - sample $3(9 \mathrm{~nm})$

Fig.2. Plot of $\chi^{\prime}(\omega)$ and $\chi^{\prime \prime}(\omega)$ against $f[\mathrm{GHz}]$ for sample $3(9 \mathrm{~nm})$ as a function of polarising field, $H$

Fig.3. Plot of $f_{\text {res }}$ against $\mathrm{H}$ for samples 1,2 and 3

Fig.4. Plot of $f_{\max } / f_{\text {res }}$ against $H$ for sample $3(9 \mathrm{~nm})$

Table 1

\begin{tabular}{|l|l|l|l|l|l|}
\hline Sample & $\begin{array}{l}\text { Mean } \\
\text { diameter } \\
10^{-9} \mathrm{~m}\end{array}$ & $\begin{array}{l}\mathrm{H}_{\mathrm{A}} \\
\mathrm{kAm}^{-1}\end{array}$ & $\begin{array}{c}\gamma \\
\mathrm{s}^{-1} \mathrm{~A}^{-1} \mathrm{~m}\end{array}$ & $\alpha$ & $\begin{array}{c}\tau_{O} \\
10^{-9} \mathrm{~s}\end{array}$ \\
\hline 1 & 6.4 & 20 & $2.4 \cdot 10^{5}$ & 0.174 & 1.197 \\
\hline 2 & 7.5 & 20 & $2.4 \cdot 10^{5}$ & 0.18 & 1.157 \\
\hline 3 & 9.0 & 35.8 & $2.21 \cdot 10^{5}$ & 0.16 & 0.789 \\
\hline
\end{tabular}

